\title{
Analysis of Reconfigurable Processors Using Petri Net
}

\author{
Hadis Heidari \\ Department of Computer Engineering, Razi University, Kermanshah, Iran \\ h.heidari@pgs.razi.ac.ir
}

\begin{abstract}
In this paper, we propose Petri net models for processing elements. The processing elements include: a general-purpose processor (GPP), a reconfigurable element (RE), and a hybrid element (combining a GPP with an RE). The models consist of many transitions and places. The model and associated analysis methods provide a promising tool for modeling and performance evaluation of reconfigurable processors. The model is demonstrated by considering a simple example. This paper describes the development of a reconfigurable processor; the developed system is based on the Petri net concept. Petri nets are becoming suitable as a formal model for hardware system design. Designers can use Petri net as a modeling language to perform high level analysis of complex processors designs processing chips. The simulation does with PIPEv4.1 simulator. The simulation results show that Petri net state spaces are bounded and safe and have not deadlock and the average of number tokens in first token is 0.9901 seconds. In these models, there are only 5\% errors; also the analysis time in these models is 0.016 seconds.
\end{abstract}

Index Terms - Reconfigurable computing, Petri net analysis, concurrent system.

\section{INTRODUCTION}

Reconfigurable computing has proven to be promising technology to increase the performance of certain algorithms in scienfic and engineering applications in recent years. Any application of iterative nature such as image processing, digital signal processing, bioinformatics, cryptography and software dfened radio etc; can be mapped on an FPGA by programming it with Hardware Descriptive Languages (HDLs). These applications have certain kernels containing interactions which are processed in parallel on the processing elements on an FPGA dined by the HDL programmer.

The same applications can take much longer time, when they are run on a General Purpose Processor (GPP) which processes the iterative kernels in a sequential manner. Traditionally, the grids utilize GPPs as their main processing elements. Because of incorporation of the REs in the grid network, there is need for appropriate models for these new processing elements to investigate the possibility of their utilization for compute intensive kernels of the grid applications. Many grid networks, such as TeraGrid are incorporating figenable computing resources in addition to general-purpose processors (GPPs) as processing elements and this combination offers better performance and higher flexibility. An approach to achieve high-performance with flexibility is to utilize collaboration of recofigurable computing elements in grid networks.

Petri nets (PNs) provide a graphical tool as well as a notational method for the formal specification of systems. Petri nets were first introduced in 1966 to describe concurrent systems. Every tool applied to the modeling and analysis of computer systems has its place. Several design methodologies for embedded systems based on different formal models have been developed in recent years. Example is the project Moses [1], which is based on high level Petri nets. Many processes may be described as a logical sequence of events. This has led the authors, among others, to the development and use of Petri nets as a tool for process and condition monitoring (PCM) [2-5]. Petri net provides powerful qualitative analysis and quantitive analysis for specifying behavior and an executable notation. Petri net have a place in computer systems performance assessment, ranging somewhere between analytical queuing theory and computer simulation. This is due to the nature of Petri nets and their ability to model concurrency, synchronization, mutual exclusion, conflict, and systems state more completely than analytical models but not as completely as simulations. Petri nets represent computer systems by providing a mean to abstract the basic elements of the system and its informational flow using only four fundamental components.

Reconfigurable computing is turning into a suitable technology for high-performance computing in scientific research. An appropriate Petri net model for reconfigurable elements along with general purpose processors is essential for analytical performance modeling of an application. There is need for promising models for new processing elements to investigate the possibility of their utilization for computing intensive kernels of the applications. The results of simulations can help in designing a system by saving a significant amount of time and resources.

In this work, the development of a model, based on the Petri net is proposed, we proposed theoretical model for processors using Petri net. We simulated the proposed models as part of a large network. The simulation results suggest that the total average error rate for all models is 
less than $5 \%$, also the analysis time in these models is 0.016 seconds.

The remainder of the paper is organized as follows: Section II presents related research. Section III introduces the related terminology. In section IV the proposed models for processing components are presented. Section $\mathrm{V}$ shows the simulation results. Finally, the conclusion is provided in Section VI.

\section{RELATED WORKS}

In this section, we discuss some related work for the modeling and simulation of reconfigurable processors.

In [6] an analytical model was proposed for reconfigurable processors using the queuing theory. The results show that the main limitation of such a system is the reconfigurable time. The model, however, does not take into account the modeling of memory modules which must be considered in real scenarios. Some developed abstract model for a reconfigurable computer, [7] focused on the performance model for runtime reconfigurable hardware accelerators. In a theoretical analysis, the factors such as speedup, communication and configuration overheads are considered, but this model does not consider the utilization of the accelerator in a network perspective. In [8] was proposed an abstract model for a reconfigurable computer which utilized both the GPP and the RE. In [9] a modeling for multiprocessor system using Petri net proposed, and [10] focused a model for a system using generalized stochastic Petri nets.

\section{DEFINITION AND CONCEPTS}

Petri net components are place, transition, arcs, and token. Places are represented graphically as a circle, transitions as a bar, arcs as directed line segments, and tokens as dots (Figure 1). Places are used to represent possible system components and their state. For example, a disk drive could be represented using a place, as could a program or other resource. Transitions are used to describe events that may result in different system states. For example, the action of reading an item from a disk drive or the action of writing an item to a disk drive could be modeled as separate transitions. Arcs represent the relationships that exist between the transitions and places. For example, disk read requests may be put in one place, and that place may be connected to the transition, removing an item from a disk thus indicating that this place is related to the transition.

Arcs provide a path for the activation of a transition; tokens are used to define the state of the Petri net. Tokens in the basic Petri net model are non-descriptive markers, which are stored in places and are used in defining Petri net marking [11].
Token

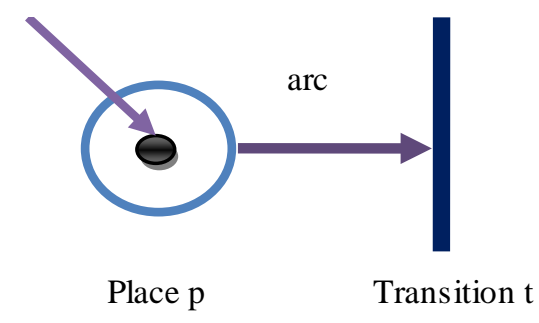

Figure 1: Basic Petri net Component

The marking of a Petri net place by the placement of a token can be viewed as the statement of the condition of the place. For example, a simple Petri net with only one place and one transition is depicted in the Figure 2. The place is connected to the transition by an arc, and the transition is likewise connected to the place by a second arc. The former arc is an input arc, while the latter arc is an output arc. The placement of a token represents the active marking of the Petri net state. The Petri net shown in Figure 2 represents a net that will continue to cycle forever. A Petri net is shown as a five tuple, $\mathrm{M}=(\mathrm{P}, \mathrm{T}, \mathrm{I}$, $\mathrm{O}, \mathrm{MP})$, where $\mathrm{P}$ portrays a set of places, $\mathrm{P}=\{\mathrm{p} 1, \mathrm{p} 2, \ldots$, $\mathrm{pn}\}$, with one place for each circle in the Petri net graph; $\mathrm{T}$ portrays a set of transitions, $\mathrm{T}=\{\mathrm{t} 1, \mathrm{t} 2, \ldots, \mathrm{tm}\}$, with one place for each bar in the Petri net graph; I shows sets of input functions for all transitions and represents mapping places to transitions; $\mathrm{O}$ shows sets of output functions for all transitions and represents mapping transitions to places and MP portrays the marking of places with tokens. The initial marking is referred to as MP0. For example, the Petri net graph depicted in Figure 3 can be represented using $\mathrm{P}=\{\mathrm{p} 1, \mathrm{p} 2, \mathrm{p} 3, \mathrm{p} 4\}, \mathrm{T}=\{\mathrm{t} 1$, $\mathrm{t} 2, \mathrm{t} 3, \mathrm{t} 4\}, \mathrm{I}(\mathrm{t} 2)=\{\mathrm{p} 4\}, \mathrm{I}(\mathrm{t} 3)=\{\mathrm{p} 4\}, \mathrm{I}(\mathrm{t} 5)=\{\mathrm{p} 1, \mathrm{p} 2\}, \mathrm{O}$ $(\mathrm{t} 1)=\{\mathrm{p} 1\}, \mathrm{O}(\mathrm{t} 2)=\{\mathrm{p} 2\}, \mathrm{O}(\mathrm{t} 3)=\{\mathrm{t} 4\}, \mathrm{O}(\mathrm{t} 5)=\{\mathrm{p} 2\}$, and $\mathrm{MP}=(0,0,0,0,0)$. Occam is a programming language based on communication sequential process concurrent computation model [12].

$\mathrm{t} 1$

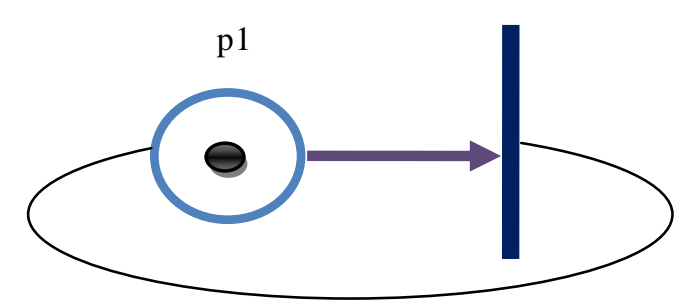

Figure 2: Example Perpetual Motion Petri net 


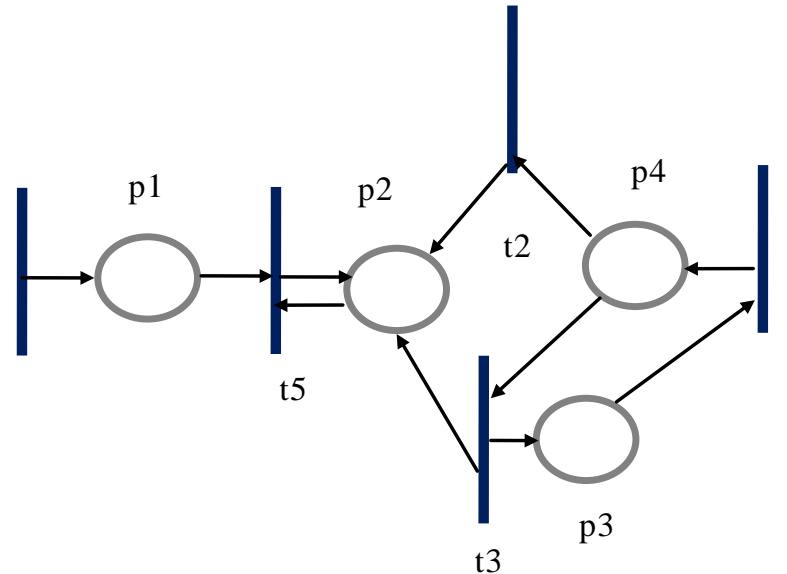

Figure 3: Example of Petri net Graph

In this section, some basic definitions and notations of ordinary $\mathrm{PN}$ are described whereas a PN is known as ordinary when all of its arc weights are 1's. The related terminology and notations are mostly taken from $[13,14]$.

Definition 1 (Petri net). A Petri net PN, is a five tuple, $\mathrm{PN}=(\mathrm{P}, \mathrm{T}, \mathrm{I}, \mathrm{O}, \mathrm{M} 0)$ where $\mathrm{P}=\{\mathrm{p} 1, \mathrm{p} 2, \ldots, \mathrm{P}|\mathrm{P}|\}$ is $\mathrm{a}$ finite set of places, $|\mathrm{P}|>0 ; \mathrm{T}=\{\mathrm{t} 1, \mathrm{t} 2, \ldots, \mathrm{t}|\mathrm{T}|\}$ is a finite set of transitions, $|\mathrm{T}|>0$; I : T P is the input function which is a mapping from transitions to the sets of their input places; $\mathrm{O}: \mathrm{T} \mathrm{P}$ is the output function which is a mapping from transitions to the sets of their output places; where $\mathrm{P} T=$ and $\mathrm{P} T$. For a transition tj $\mathrm{T}$, $\mathrm{I}(\mathrm{tj})$ and $\mathrm{O}(\mathrm{tj})$ represent the sets of input and output places of tj respectively. A place pi $P$ is the input place of a transition $\mathrm{tj}$ if pi $\mathrm{I}(\mathrm{tj})$ and the output place of $\mathrm{tj}$ if $\mathrm{pi}$ $\mathrm{O}(\mathrm{tj})$. The input and output functions can be extended to map the set of places $\mathrm{P}$ into the set of transitions $\mathrm{T}$ such as I: P T and O: P T. Then, I (pi) represent the set of input transitions of place pi $\mathrm{P}$ and $\mathrm{O}$ (pi) represents the set of output transitions of place pi $\mathrm{P}$. The structure of a $\mathrm{PN}$ is defined by a set of places, a set of transitions, an input function and an output function. A PN structure without M0 is denoted by $\mathrm{N}=(\mathrm{P}, \mathrm{T}, \mathrm{I}, \mathrm{O})$. A PN structure $\mathrm{N}$ is said to be strongly connected if and only if every node $\mathrm{xj}$ $\mathrm{P} \quad \mathrm{T}$ is reachable from every other node xi $\mathrm{P} \quad \mathrm{T}$ by $\mathrm{a}$ directed path. A PN structure $\mathrm{N}$ is said to be self-loopfree or pure if and only if $\mathrm{tj} \mathrm{T}, \mathrm{I}(\mathrm{tj}) \mathrm{O}(\mathrm{tj})=$, i.e., no place can be both an input and an output of the same transition. A marking is a function M: P N (non-negative integers) and initial marking is denoted by M0. A PN with given initial marking is denoted by a pair $(\mathrm{N}$, $\mathrm{M} 0$ ). The set of all reachable markings from $\mathrm{MO}$ is denoted by $\mathrm{R}$ (M0) which is a definite set of markings of PN such that, if $\mathrm{Mk} \mathrm{R}$ (M0).

Definition 2 (Firing rule). The firing rule identifies the transition enabling and the change of marking. Let $\mathrm{M}$ (pi) be the number of tokens in place pi, then for $t j \mathrm{~T}$; $t \mathrm{j}$ is enabled under marking $M$ if and only if pi $I(t j): M(p i)$ 1. The change of marking $M$ to M0 by firing the enabled transition $t_{j}$ is denoted by $M\left[t j>M^{\prime}\right.$ and defined for each place pi P by:

$$
M^{\prime}\left(p_{i}\right)= \begin{cases}M\left(p_{i}\right)-1 \text { for every } p_{i} & I\left(t_{j}\right), \\ M\left(p_{i}\right)+1 \text { for every } p_{i} & O\left(t_{j}\right) \\ M\left(p_{i}\right) \text { otherwise } & \end{cases}
$$

Definition 3: for PN (N, $\left.M_{0}\right)$ and $M \quad R\left(M_{0}\right)$, let $t_{j}$ and $t_{1}$ are enabled at marking $M$, then $t_{j}$ and $t_{1}$ are concurrently enabled at $\mathrm{M}$ if and only if $\mathrm{M}\left(\mathrm{p}_{\mathrm{i}}\right) \quad \mathrm{O}\left(\mathrm{p}_{\mathrm{i}}\right)$ for $p_{i} \quad I\left(t_{j}\right) \quad I\left(t_{1}\right)$. It is noteworthy, if $M$ enables both $t_{j}$ and $t_{1}$ then it is not necessarily true that $t_{j}$ and $t_{1}$ are concurrently enabled at M. For I $\left(t_{j}\right) \quad I\left(t_{1}\right)=$, any marking which enables $t_{j}$ and $t_{1}$, enables them concurrently.

\section{MODELING OF RECONFIGURABLE PROCESSOR USING PET RI NET}

In this section, we describe our proposed models for reconfigurable processors. Recfogurable computing provides much moreflexibility than Application -Specific Integrated Circuits (ASICs) and much more performance than General-Purpose Processors (GPPs). GPPs, reconfigurable elements (RE) and hybrid (integration of GPPs and REs) elements are the main processing elements.

\section{A. GPP Petri net Model}

We have a central processing unit and a memory module. The steps in the GPP Petri net model are:

1. The tasks are waiting in the CPU queue

2. The tasks are serviced by CPU

3. The tasks are going to the memory queue

4. The tasks are waiting in the memory queue

5. The tasks are serviced by memory

6. The tasks are going to the CPU queue

This operation is repeated. $\mathrm{PN}$ model of reconfigurable processor for a GPP is shown in Figure 4. Corresponding notations are described in table I and table II.

\section{B.Proposed Model for Reconfigurable Processing Elements}

The proposed model for a RE consists of a reconfigurable processor, memory module, and a reconfigurable module. The steps in the reconfiguration elements could be summarized as follows:

1. The tasks are waiting in the reconfigurable processor queue

2. The tasks are waiting in the reconfigurable module queue

3. The tasks are serviced by reconfigurable process or

4. The tasks are serviced by reconfigurable module

5 . The tasks are going to the memory queue

6 . The tasks are wait ing in the memory modules queue

7. The tasks are serviced by memory modules

8. The tasks are going to reconfigurable processor queue or is going to reconfigurable module

This operation is repeated. PN model of reconfigurable processor for a RE is shown in Figure 5. 
Corresponding notations are described in table III and table IV.

\section{C.Proposed Model for a Hybrid Processing Element}

The proposed model for a hybrid processing element consists of a GPP and a RE. The steps in the reconfiguration elements could be summarized as follows:

1. The tasks are waiting in the CPU queue

2. The tasks are waiting in the reconfigurable processor queue

3. The tasks are waiting in the reconfigurable module queue
4. The tasks are serviced by CPU

5. The tasks are services by reconfigurable processor

6. The tasks are services by reconfigurable module

7. The tasks are going to the memory queue

8. The tasks are waiting in the memory modules queue

9. The tasks are serviced by memory modules

10. The tasks are going to CPU queue or reconfigurable processor queue or reconfigurable module queue.

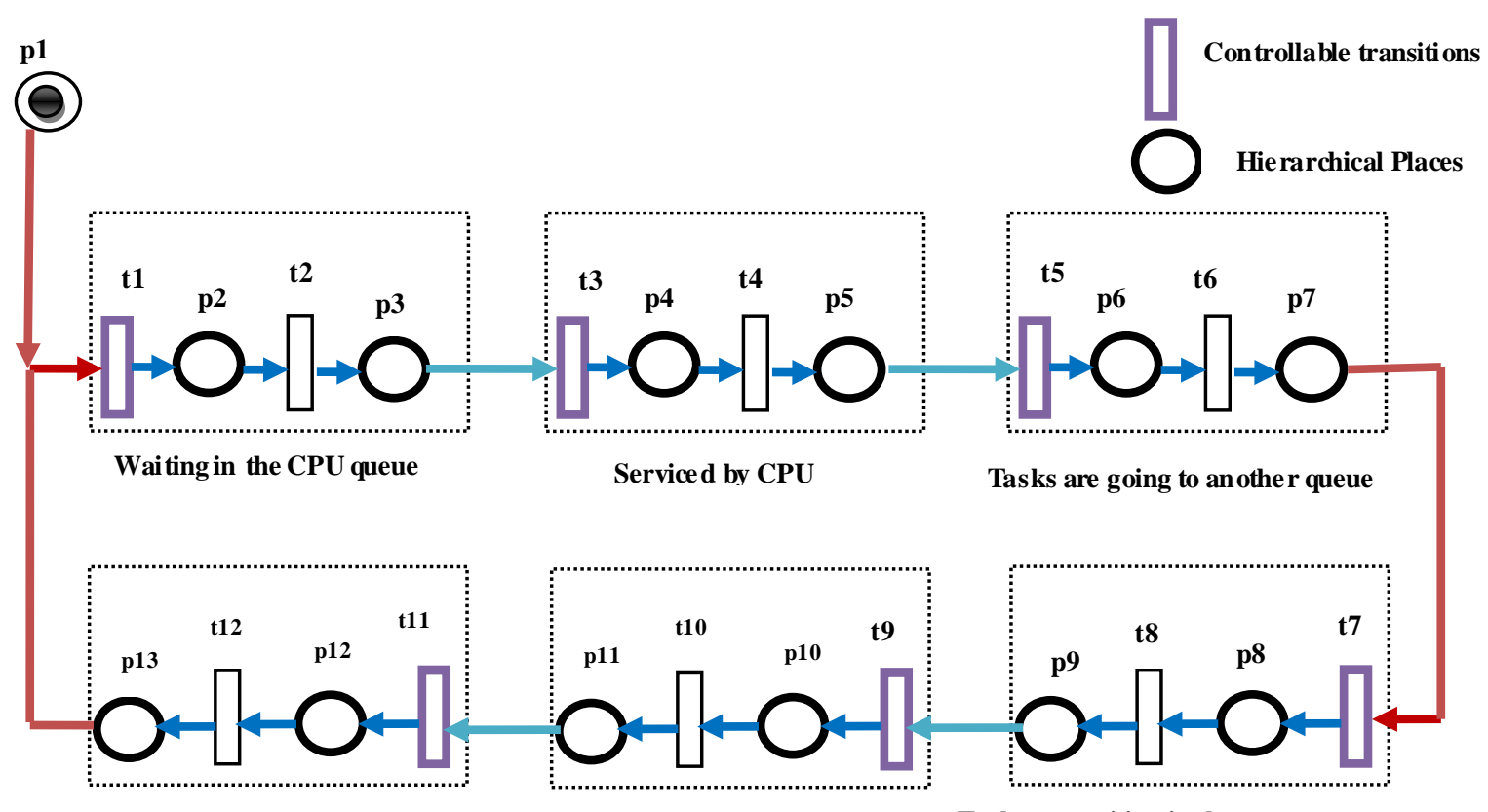

Tasks are going to CPU queue

Tasks are serviced by memory

Tasks are waiting in the memory queue

Figure 4: Petri net Model for a GPP

TABLE I. NOTATION FOR THE PNOF THE FIGURE 4

\begin{tabular}{|c|l|}
\hline Place & \multicolumn{1}{c|}{ Description } \\
\hline p1 & Tasks entered in CPU queue \\
\hline p2 & Waiting in the CPU queue \\
\hline p3 & Waiting in the CPU queue completed \\
\hline p4 & Serviced by CPU \\
\hline p5 & Serviced by CPU completed \\
\hline p6 & Tasks are going to another queue \\
\hline p7 & Tasks are going to another queue completed \\
\hline p8 & Tasks are waiting in the memory queue \\
\hline p9 & Tasks are waiting in the memory queue completed \\
\hline p10 & Tasks are serviced by memory \\
\hline p11 & Tasks are serviced by memory completed \\
\hline p12 & Tasks are going to CPU queue \\
\hline p13 & Tasks are going to CPU queue completed \\
\hline
\end{tabular}




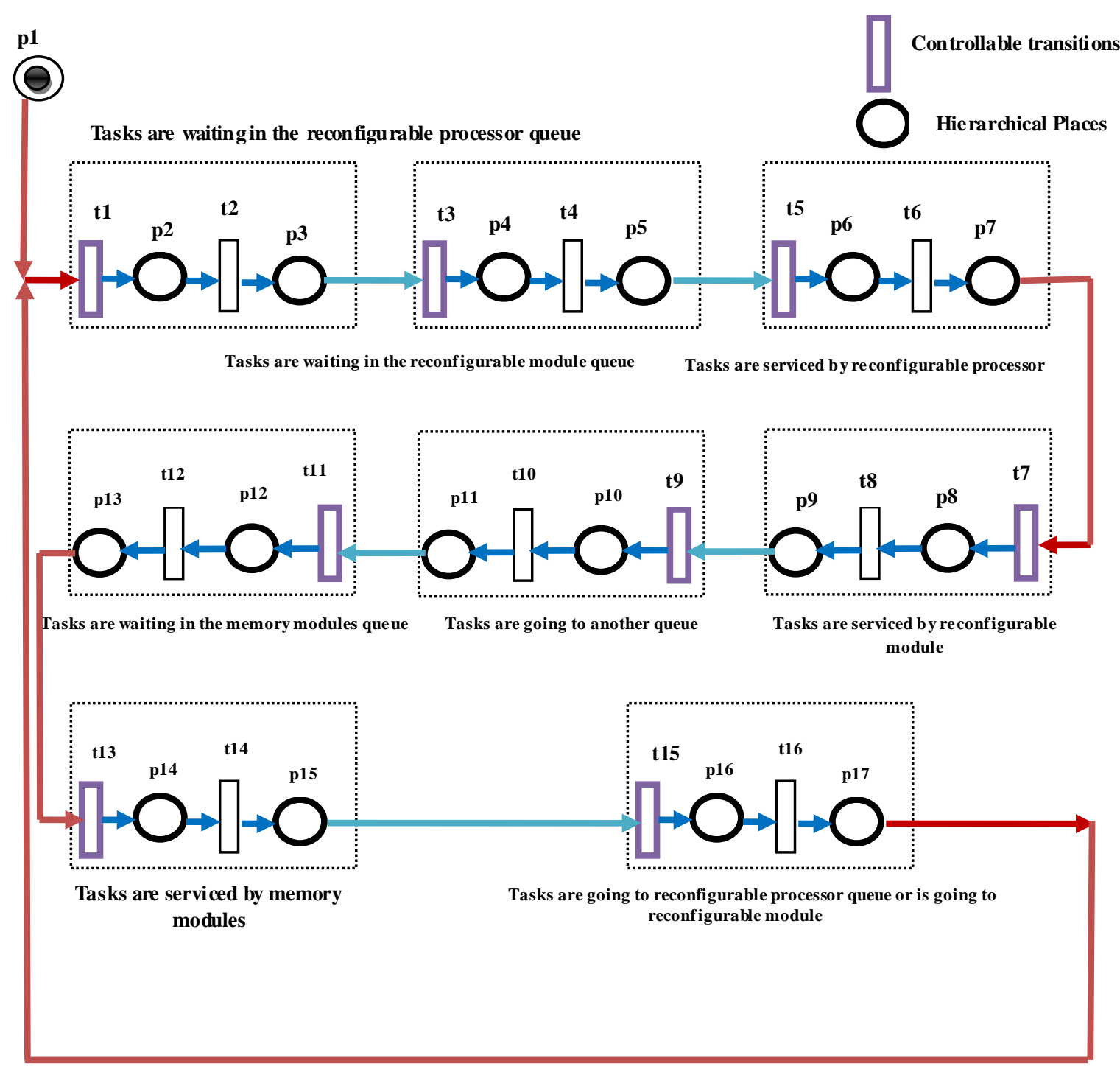

Figure 5: Petri net Model for a RE

This operation is repeated. PN model of reconfigurable processor for a hybrid processing element is shown in Figure 6. Corresponding notations are described in table V and table VI. Colored Petri nets add another dimension to tokens as well as to selection criteria used in determining firing by the addition of different token types.
Tokens can represent different functions. We can use different tokens to represent operating system calls or different classes of jobs. These different tokens can then be used to determine which transition of multiple transitions available operates.

TABLE II. NOTATION ABOUT TRANSITION OF THE FIGURE 4

\begin{tabular}{|c|l|}
\hline Transition & \multicolumn{1}{|c|}{ Description } \\
\hline $\mathrm{t} 1$ & Start entered tasks in the CPU queue \\
\hline $\mathrm{t} 2$ & End waiting in the CPU queue \\
\hline $\mathrm{t} 3$ & Start serviced by CPU \\
\hline $\mathrm{t} 4$ & End serviced by CPU \\
\hline $\mathrm{t} 5$ & Start tasks are going to another queue \\
\hline $\mathrm{t} 6$ & End tasks are going to another queue \\
\hline $\mathrm{t} 7$ & Start tasks are waiting in the memory queue \\
\hline $\mathrm{t} 8$ & End tasks are waiting in the memory queue \\
\hline $\mathrm{t} 9$ & Start tasks are serviced by memory \\
\hline $\mathrm{t} 10$ & End tasks are serviced by memory \\
\hline $\mathrm{t} 11$ & Start tasks are going to CPU queue \\
\hline $\mathrm{t} 12$ & End tasks are going to CPU queue \\
\hline
\end{tabular}




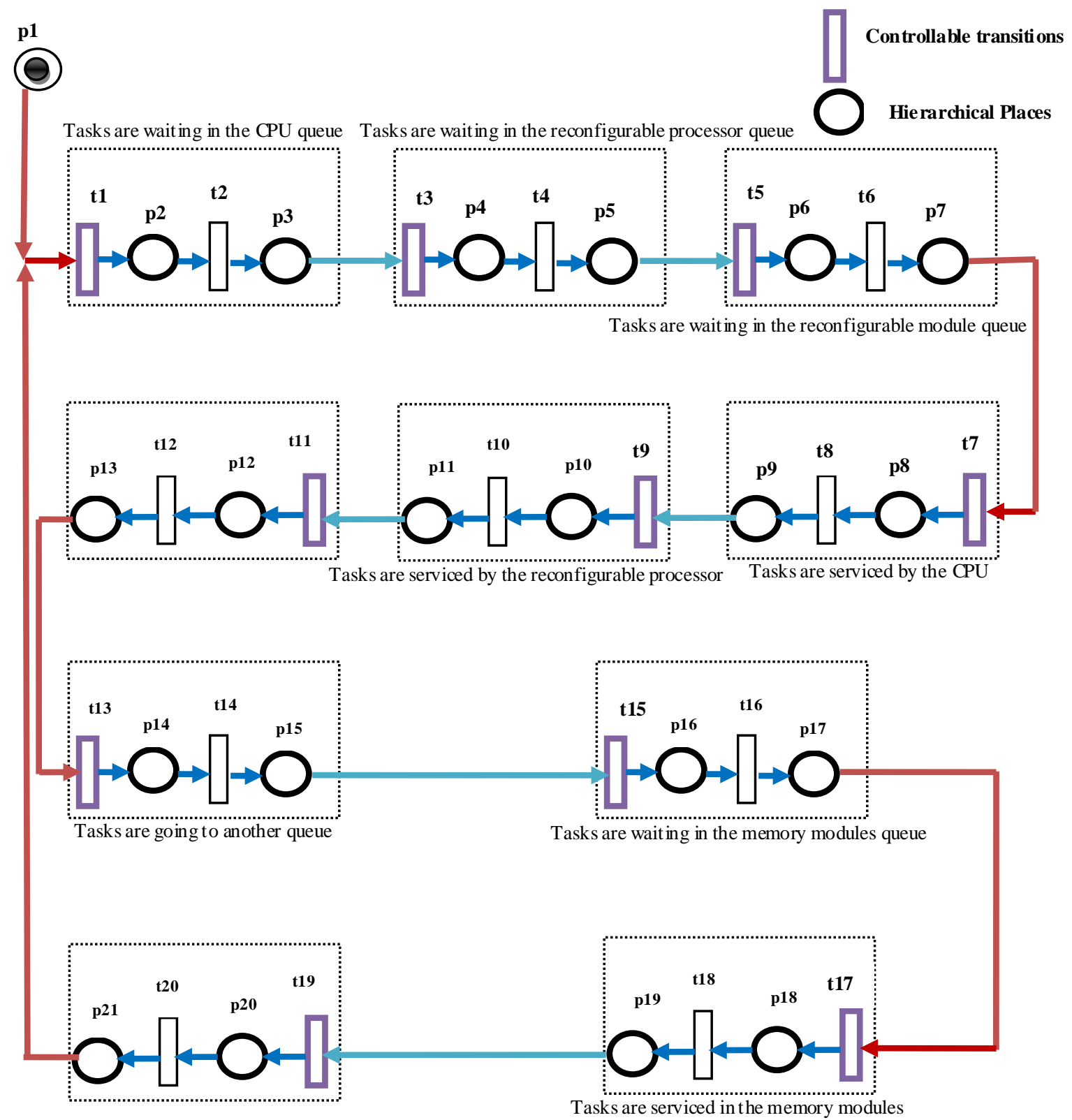

Figure 6: Petri net Model for a hybrid processing element

TABLE III. NOTATION FOR THE PN OF THE FIgURE 5

\begin{tabular}{|l|l|}
\hline Places & \multicolumn{1}{|c|}{ Description } \\
\hline p1 & Tasks entered in the reconfigurable processor queue \\
\hline p2 & Tasks are waiting in the reconfigurable processor queue \\
\hline p3 & Tasks are waiting in the reconf igurable processor queue completed \\
\hline p4 & Tasks are waiting in the reconf igurable module queue \\
\hline p5 & Tasks are waiting in the reconfigurable module queue completed \\
\hline p6 & Tasks are served by reconfigurable processor \\
\hline p7 & Tasks are served by reconfigurable processor completed \\
\hline p8 & Tasks are served by reconfigurable module \\
\hline p9 & Tasks are served by reconfigurable module completed \\
\hline p10 & Tasks are going to another queue \\
\hline p11 & Tasks are going to another queue completed \\
\hline p12 & Tasks are waiting in the memory module queue \\
\hline p13 & Tasks are waiting in the memory module queue completed \\
\hline p14 & Tasks are serviced by memory modules \\
\hline p15 & Tasks are serviced by memory modules completed \\
\hline p16 & Tasks are going to reconfigurable processor queue or is going to reconfigurable module \\
\hline p17 & Tasks are going to reconfigurable processor queue or is going to reconfigurable module completed \\
\hline
\end{tabular}


TABLE IV. NOTATION ABOUT TRANSITION OF THE FIGURE 5

\begin{tabular}{|c|l|}
\hline Transition & \\
\hline t1 & Start entered tasks in the reconfigurable processor queue \\
\hline t2 & End tasks are waiting in the reconfigurable processor queue \\
\hline t3 & Start tasks are waiting in the reconfigurable module queue \\
\hline t4 & End tasks are waiting in the reconfigurable module queue \\
\hline t5 & Start tasks are serviced by reconfigur able processor \\
\hline t6 & End tasks are serviced by reconfigurable processor \\
\hline t7 & Start tasks are serviced by reconfigurable module \\
\hline t8 & End tasks are serviced by reconfigurable module \\
\hline t9 & Start tasks are going to another queue \\
\hline t10 & End tasks are going to another queue \\
\hline t11 & Start tasks are waiting in the memory modules queue \\
\hline t12 & End tasks are waiting in the memory modules queue \\
\hline t13 & Start tasks are serviced by memory modules \\
\hline t14 & End tasks are serviced by memory modules \\
\hline t15 & Start tasks are going to reconfigurable processor queue or is going to reconfigurable module \\
\hline t16 & End tasks are going to reconfigurable processor queue or is going to reconfigurable module \\
\hline
\end{tabular}

TABLE V. NOTATION ABOUT TRANSITION OF THE FIGURE 6

\begin{tabular}{|c|c|}
\hline Places & Description \\
\hline $\mathrm{p} 1$ & Tasks entered in the CPU queue \\
\hline p2 & Tasks are waiting in CPU queue \\
\hline p3 & Tasks are waiting in the CPU queue completed \\
\hline $\mathrm{p} 4$ & Tasks are waiting in the reconfigurable processor queue \\
\hline p5 & Tasks are waiting in the reconfigurable processor queue completed \\
\hline p6 & Tasks are waiting in the reconfigurable module queue \\
\hline p7 & Tasks are waiting in the reconfigurable module queue completed \\
\hline p8 & Tasks are served by CPU \\
\hline p9 & Tasks are served by CPU completed \\
\hline $\mathrm{p} 10$ & Tasks are served by reconfigurable processor \\
\hline $\mathrm{p} 11$ & Tasks are served by reconfigurable processor completed \\
\hline $\mathrm{p} 12$ & Tasks are served by reconfigurable module \\
\hline $\mathrm{p} 13$ & Tasks are served by reconfigurable module completed \\
\hline $\mathrm{p} 14$ & Tasks are going to another queue \\
\hline p15 & Tasks are going to another queue completed \\
\hline p16 & Tasks are waiting in the memory module queue \\
\hline p17 & Tasks are waiting in the memory module queue completed \\
\hline p18 & Tasks are serviced by memory modules \\
\hline $\mathrm{p} 19$ & Tasks are serviced by memory modules completed \\
\hline p20 & Tasks are going to CPU queue or reconfigurable processor queue or is going to reconfigurable module queue \\
\hline p21 & Tasks are going to CPU queue or reconfigurable processor queue or is going to reconfigurable module queue completed \\
\hline
\end{tabular}

TABLE VI. NOTATION ABOUT TRANSITION OF THE FIGURE 6

\begin{tabular}{|c|l|}
\hline Transition & \\
\hline t1 & Start entered tasks in the CPU queue \\
\hline t2 & End tasks are waiting in the CPU queue \\
\hline t3 & Start tasks are waiting in the reconfigurable processor que ue \\
\hline t4 & End tasks are waiting in the reconfigurable processor que ue \\
\hline t5 & Start tasks are waiting in the reconfigurable module queue \\
\hline t6 & End tasks are waiting in the reconfigurable module queue \\
\hline t7 & Start tasks are serviced by CPU \\
\hline t8 & End tasks are serviced by CPU \\
\hline t9 & Start tasks are serviced by reconfigurable processor \\
\hline t10 & End tasks are serviced by reconfigurable processor \\
\hline t11 & Start tasks are serviced by reconfigurable module \\
\hline t12 & End tasks are serviced by reconfigurable module \\
\hline t13 & Start tasks are going to another queue \\
\hline t14 & End tasks are going to another queue \\
\hline t15 & Start tasks are waiting in the memory modules queue \\
\hline t16 & End tasks are waiting in the memory modules queue \\
\hline t17 & Start tasks are serviced by memory modules \\
\hline t18 & End tasks are serviced by memory modules \\
\hline t19 & Start tasks are going to CPU queue or reconfigurable processor queue or is going to reconfigurable module \\
\hline t20 & End tasks are going to CPU queue or reconfigurable processor queue or is going to reconfigurable module \\
\hline
\end{tabular}




\section{SIMULATION AND EXPERIMENT}

The open queuing model in our previous work was validated by computing the average response time results using Maple v.13 analytically and compared with those generated experimentally using $\mathrm{OMNeT}++$ simulator. The service rate of GPP ( $\mu_{\mathrm{GPP}}$ ) varies between 1 and 80 , whereas the speedup of RE is taken as 5 , Therefore, the service rate of $\mathrm{RE}$ was set to $5 x \mu_{\mathrm{GPP}}$. Generally, a $\mathrm{RE}$ requires a reconfiguration for a set of incoming tasks. In our experiments we assumed that, for each 1000 incoming tasks, one recofiguration i s needed. The total average error for GPP and RE models for all arrival rates is $2.86 \%$ and $2.67 \%$, respectively. These percentage values of error were calculated by dividing total sum of error rates for each model by the respective arrival rates. The simulation results for average response time for all two models are in accordance with the average response time results computed analytically within a range of less than $5 \%$ relative error.

The proposed models were validated by computing the analysis time using PIPEv4.1 simulator. The simulation result shows that Petri net state spaces are bounded and safe and have not deadlock. The average of number tokens in first token is 0.9901 seconds. In these models, there are only 5\% errors; also the analysis time in these models is 0.016 seconds. The Petri net invariant analysis results are provided in the table VII.

TABLE VII. PETRI NET INVARIENT ANALYSIS RESULTS

\begin{tabular}{|c|c|c|c|c|c|c|c|c|c|c|c|c|c|c|c|c|} 
T0 & $\mathbf{T 1}$ & $\mathbf{T 2}$ & $\mathbf{T 3}$ & $\mathbf{T 4}$ & $\mathbf{T 5}$ & $\mathbf{T 6}$ & $\mathbf{T}$ & $\mathbf{T 8}$ & $\mathbf{T 9}$ & $\mathbf{T 1 0}$ & $\mathbf{T 1 1}$ & $\mathbf{T 1 2}$ & $\mathbf{T 1 3}$ & $\mathbf{T 1 4}$ & $\mathbf{T 1 5}$ & $\mathbf{T 1 6}$ \\
\hline 0 & 0 & 0 & 0 & 0 & 0 & 1 & 0 & 0 & 0 & 0 & 0 & 0 & 0 & 0 & 0 & 0 \\
\hline 0 & 0 & 0 & 0 & 0 & 0 & 0 & 1 & 0 & 0 & 0 & 0 & 0 & 0 & 0 & 0 & 0 \\
\hline 0 & 0 & 0 & 0 & 0 & 0 & 0 & 0 & 0 & 0 & 1 & 0 & 0 & 0 & 0 & 0 & 0 \\
\hline 0 & 0 & 0 & 0 & 0 & 0 & 0 & 0 & 0 & 0 & 0 & 0 & 1 & 0 & 0 & 0 & 0 \\
\hline 0 & 0 & 0 & 0 & 0 & 0 & 0 & 0 & 0 & 0 & 0 & 0 & 0 & 0 & 1 & 0 & 0 \\
\hline
\end{tabular}

In our models, there are sixteen places, $\mathrm{p} 0$ is first place, and the average number of tokens in each place is depicted in bellow. The simulation results are depicted in the table VIII. The average number of tokens in first place is 0.9901 , the average number of tokens in another places is zero. The simulation result in our models is shown that there is $95 \%$ confidence interval.

TABLE VIII. PETRI NET INVARIENT ANALYSIS RESULTS

\begin{tabular}{|c|c|c|}
\hline Place & $\begin{array}{c}\text { Average number of } \\
\text { tokens }\end{array}$ & $\begin{array}{c}\text { 95\% confidence } \\
\text { interval }(+/-)\end{array}$ \\
\hline P0 & 0.9901 & 0 \\
\hline P1 & 0 & 0 \\
\hline P2 & 0 & 0 \\
\hline P3 & 0 & 0 \\
\hline P4 & 0 & 0 \\
\hline P5 & 0 & 0 \\
\hline P6 & 0 & 0 \\
\hline P7 & 0 & 0 \\
\hline P8 & 0 & 0 \\
\hline P9 & 0 & 0 \\
\hline P10 & 0 & 0 \\
\hline
\end{tabular}

\begin{tabular}{|l|l|l|}
\hline P11 & 0 & 0 \\
\hline P12 & 0 & 0 \\
\hline P13 & 0 & 0 \\
\hline P14 & 0 & 0 \\
\hline P15 & 0 & 0 \\
\hline
\end{tabular}

\section{CONCLUSIONS}

In this paper, based on Petri net model, we proposed Petri net models for the processing element. The proposed models can be useful in implementing the real grid networks with $\mathrm{RE}$ as one of the processing elements. In our models, the average of number tokens in first token is 0.9901 seconds and there are only $5 \%$ errors, also the analysis time in these models is 0.016 seconds.

\section{REFERENCES}

[1] “The Moses Project”. http://www.tik.ee.ethz.ch/ moses/

[2] S.K. Yang, T.S. Liu, A Petri-net approach to early failure detection and isolation for preventive maintenance, Quality and Reliability Engineering International 14 (1998) 319-330.

[3] P.W. Prickett, R.I. Grosvenor, Non-sensor based machine tool and cutting process condition monitoring, International Journal of COMADEM 2 (1) (1999) 31-37.

[4] A.D. Jennings, D. Nowatschek, P.W. Prickett, V.R. Kennedy, J.R. Turner, R.I. Grosvenor, Petri net Based Process Monitoring, in: Proceedings of COMADEM, Houston, USA, 3-8 December 2000, MFPT Society,, Virginia, 2000.

[5] P. Prickett, R. Grosvenor, A Petri-net based machine tool failure diagnosis system, Journal of Quality in Maintenance Engineering 18 (30) (1995) 47-57.

[6] F. Lotfifar, S. Shahhiseini, "Performance Modeling of Partially Reconfigurable Computing Systems”, In Proc. of the 6th IEEE/ACS International Conference Systems and Applications (AICCSA), pp. 94-99, 2008.

[7] G. Wang, et al., "A Performance Model for RunTime Reconfigurable Hardware Accelerator", In Proc. of the 8th International Symposium on Advanced Parallel Processing Technologies (APPT), pp. 54-66, 2009.

[8] U. Vishkin, et al., "Handbook of Parallel Computing: Models, Algorithms and Applications", in the chapter "A Hierarchical Performance Model for Reconfigurable Computers”, CRC Press, 2007.

[9] Tabak, D. Lewis, "Petri net representation of decision models”, IEEE Trans, on S. M. C, pp 812818, 1989.

[10] M. A. Marsan et al., "Modeling with generalized stochastic Petri nets", in Wiley Series in Parallel Computing. New York: Wiley, 1995.

[11] Computer systems performance evaluation and prediction, P.J. Fourier and H.E. Michel, Digital press 2003. 
[12] C. A. R, Hoare, Communication Sequential Processes, Prentice-Hall, 1985.

[13] T. Murata, Petri net analysis and application, Proceedings of IEEE 77 (1989) 541-580.

[14] J.L. Peterson, Petri net Theory and the Modeling of Systems, Prentice-Hall, Englewood Cliffs, NJ, 1981.

\section{AUTHO RS’ ProfiLe}

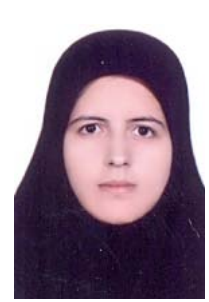

Hadis Heidari is currently a M.Sc. student in Razi University of Kermanshah, Iran. She obtained her B.S. in Computer Engineering from the same University in 2011. She was the first rank of students in Computer Engineering in the Razi University. Her research interests are in the fields of operating systems, computer networks, reconfigurable computing and image processing. 